\title{
Inverse Modelling and Medical Imaging Applied to Biomechanical Research
}

\author{
Carlos Llopis-Albert*1, Francisco Rubio ${ }^{2}$, José M. Merigó ${ }^{3,4}$, Francisco Valero ${ }^{2}$ \\ ${ }^{1}$ Departamento de Ingeniería Mecánica y de Materiales, Universitat Politècnica de València, Spain \\ ${ }^{2}$ Centro de Investigación en Ingeniería Mecánica, Universitat Politècnica de València, Spain
}

${ }^{3}$ Department of Management Control and Information Systems, School of Economics and Business, University of Chile, Av. Diagonal Paraguay 257,8330015 Santiago, Chile

${ }^{4}$ King Saud University, Riyadh, Saudi Arabia

Received: April 29, 2018; Published: May 14, 2018

*Corresponding author: Carlos Llopis Albert, Departamento de Ingeniería Mecánica y de Materiales, Universitat Politècnica de València - Camino de Vera s/n, 46022, Spain

Abstract

The prediction of heterogeneous material properties in biomedical applications is becoming a major problem in cases with complex microstructures and multiphase materials. Several branches in medicine require accurate measurements, which can be achieved by means of medical imaging. They are non-destructive techniques to visualize the inside features of solid objects and for obtaining digital information on their three-dimensional geometries and properties. Therefore, for relating the observed measurements to unknown physical parameters, through medical imaging reconstruction, inverse modelling approaches are advisable.

Keywords: Inverse Modelling; Medical Imaging; Biomechanics; Finite Element Method; Heterogeneity; Uncertainty

Abbreviations: CT: Computed Tomography; MR: Magnetic Resonance; DIC: Digital Image Correlation

\section{Introduction}

Inverse model approaches are used in a wide range of applications which include the analysing biomechanical implants, the derivation of material properties from ultrasound data (e.g. elastic properties of human tissues), the reconstruction of an image in X-ray computed tomography (CT) or of a magnetic resonance (MR), and in the fluorescence microscopy technique used for studying living cells. For instance, optical methods are applied for deriving the local deformation of soft tissue and bone in response to a loading experiment and then to derive the biological material properties from them. Despite the inverse modelling has been exhaustively used in many research fields, these approaches still have a great potential for improvement in biomedical applications. It applies mathematical techniques and develops computational algorithms to reduce uncertainty and provide accurate predictions by taking profit of the information provided by medical imaging. These techniques use actual results of measurements of observable parameters to estimate the actual values of the model parameters by means of a mathematical optimisation criterion [1].

Then the available measured data together with prior knowledge about the physical phenomena are used for obtaining estimates of the desired variables, which may vary both spatially and temporally. The reproduction of a diagnosis image entails the reproduction of both the signal contained in the image and the noise disturbing that signal. Additionally, local tissue varying stiffness, strength and anisotropy prevent the achievement of reliable estimates. In this way, the use of numerical simulation together with medical imaging and experiments lead the material properties to be adjusted until the model matches the experiment. For that purpose, the iterative optimization procedure of inverse methods usually combines Finite Element Models, which deal with the underlying physical processes, with the information provided by optical full-field measurements. For example, inverse algorithms are applied to the assessment of biomechanical properties of human tissue subject to tension or for tissue fracture, which is one of the major challenges in biomechanics.

Then the strain and stress maps allow to characterize the mechanical heterogeneity of the parameters and the subsequent mechanical response. Several drawbacks may arise when using an inverse model. On the one hand, it should be considered that these approaches are not well-posed problems, which means that a 
solution exists, it is unique and its behaviour changes continuously with the initial conditions. On the other hand, the definition of the conceptual model and the collection of experimental measurements present many practical and theoretical challenges, which lead to numerical errors and inaccuracies. The computational cost of an inverse model is also a key issue, since during the iterative optimization problem for constraining stochastic simulations to data the algorithm is repeatedly called with different material parameters. Therefore, the optimization procedure must be solved in a quickly and reliably way. To overcome these disadvantages several methods to reduce the computational cost may be applied, which can be broadly classified in three groups.

A first solution is to reduce the cost of a posterior evaluation (i.e., the cost of a forward simulation) by means of surrogate models, reduced-order models, multigrid and multiscale techniques, and stochastic spectral methods. Secondly, the dimension of the input space can be decreased, via truncated expansions, coarse grids, and parameter-space reductions. That is, the complexity of the solution space can be controlled using regularization techniques, which approximate the inverse problem by a family of stable operators. The third way seeks to reduce the number of forward simulations needed to compute specific estimators by means of highly efficient sampling methods. Another factor to consider is the fact that the mechanical behaviour of biological tissue exhibits certain characteristics that may influence the mechanical response and disturb material identification, for instance, visco-elasticity and multi-scale properties, and their variability and remodelling.

For instance, the biological tissue that connects tendons to bone presents local values of the elastic moduli that can differ by as much as two orders of magnitude to match the stiff surface of bone with the soft tendon. Therefore, highly heterogeneous materials with properties varying over several orders of magnitude, and hence with gradients of a certain variable with extreme soft-tohard transitions, require an inverse model to iteratively tune the parameter field and achieve reliable results. Another important reason that encourages the use of inverse models in biomechanics is the fact of characterizing the mechanical behaviour of tissues at diverse strain levels and under dynamic loading. In addition to the experimental data and numerical models, a suitable optimisation procedure should be selected to adjust the material properties, to provide the best match with the experimental results, and to take into account matters of uniqueness of the identified material parameters.

If only a single parameter is optimised a global optimum can be easily achieved, but for complex parametrization models many different parameter sets can lead to equally good results. The issue of uniqueness can be addressed by means of stochastic approaches (e.g., using Monte Carlo simulations of equally likely parameter fields), by using a larger quantity of experimental measurements or by using a priori information, i.e., the knowledge of expected properties of the solution. This can be achieved by tracking the full-field deformation field using optical measurements or medical imaging techniques, such as the digital image correlation (DIC) method. These methods are becoming increasingly important for material identification, for instance, of biological tissues. It should also be considered that successful patient-specific results can be achieved without a precise knowledge of the underlying material properties. Such cases arise when numerical biomedical models can be loaded kinematically or when biological structures are roughly statically determinate. Examples are image-guided surgery or neurosurgery and the modelling of thin walled biological organs.

\section{Conclusion}

As a summary, inverse problems and material properties identification is a fast-growing research area for biomedical applications. New methodologies are required to tackle the shortterm challenges and future trends and we hope that this paper provides a useful contribution to this purpose and encourages researchers from different scientific disciplines to collaborate.

\section{References}

1. Bertero M, Piana M (2006) Inverse problems in biomedical imaging: modeling and methods of solution. In: Quartertones A, Formaggia L, Veneziani A (eds) Complex Systems in Biomedicine. Springer Milano pp: $1-33$.
This work is licensed under Creative Commons Attribution 4.0 License

Submission Link: https://biomedres.us/submit-manuscript.php

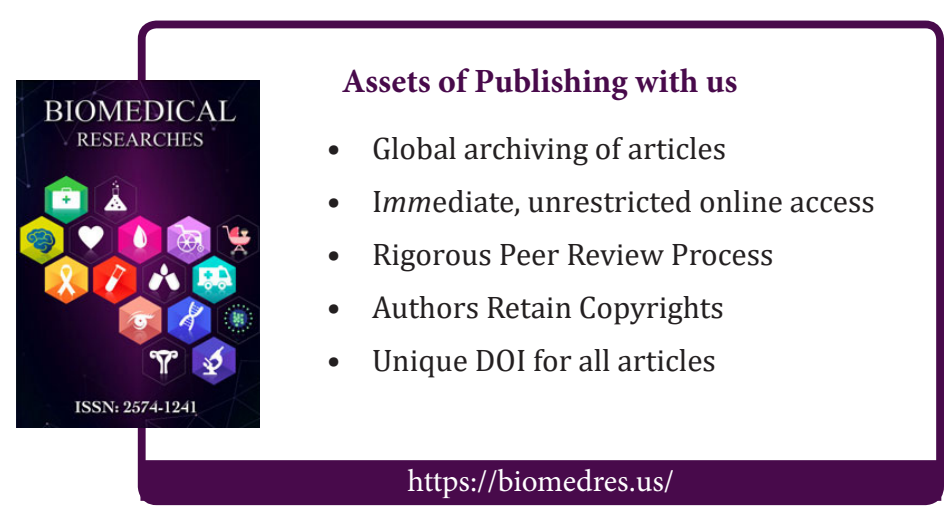

